\title{
Enhanced efficacy of IL-15-based ALT-803 superagonist complex in combination with immune checkpoint inhibitors in hematologic and metastatic mouse tumor models
}

\author{
Wenxin Xu, Warren D Marcus, Peter R Rhode*, Hing C Wong \\ From Society for Immunotherapy of Cancer 29th Annual Meeting \\ National Harbor, MD, USA. 6-9 November 2014
}

Recent advances in achieving highly durable clinical responses via inhibition of immune checkpoint molecules have revolutionized the outlook for cancer immunotherapy. However, such responses are only observed in a minority of patients, suggesting that strategies to further augment antitumor immune activity may provide additional clinical benefit. ALT-803 is an IL-15 superagonist: IL-15R $\alpha$-Fc complex capable of stimulating T cell and NK cell responses without inducing Treg activity. ALT-803 has improved pharmacokinetics and biodistribution compared to native IL-15, allowing this complex to exhibit more potent efficacy than IL-15 in various hematologic and solid tumor models. Mechanism-of-action studies showed that ALT-803 is capable of inducing both innate effector-like and adaptive $\mathrm{T}$ cell antitumor activity, suggesting that the immunostimulatory effects of ALT-803 could compliment the activity of checkpoint inhibitors to block tumor immune evasion. To assess this, we utilized a murine 5T33P myeloma model in which ALT-803 was shown to be effective at decreasing tumor burden in the bone marrow and prolonging animal survival. Treatment of mice bearing established 5T33P tumors (5/group) with $5 \mathrm{mg} / \mathrm{kg}$ anti-PD-L1 mAb also prolonged survival compared to controls $(\mathrm{P}>0.01)$, whereas treatment with 1.25 $\mathrm{mg} / \mathrm{kg}$ anti-PD-L1 $\mathrm{mAb}$ or $10 \mathrm{mg} / \mathrm{kg}$ anti-CTLA- $4 \mathrm{mAb}$ had no significant antitumor effect $(\mathrm{p}<0.05)$. Consistent with these results, 5T33P cells were found to express PD-L1 but not CD80/CD86. Treatment with sub-optimal $0.25 \mathrm{mg} / \mathrm{kg}$ anti-PD-L1 $\mathrm{mAb}$ or $50 \mu \mathrm{g} / \mathrm{kg}$ ALT-803 was ineffective at prolonging survival of 5T33P-bearing mice (median survival; PBS, 27 days; anti-PD-L1 mAb, 31 day;

Altor BioScience Corp., Miramar, FL, USA 